\title{
Accuracy Improvement of Pedestrian Dead-Reckoning Based Map Heading Constraint in GNSS-Denied Environments
}

\author{
Mohamed Shebl, Mohamed El-Tokhey, Tamer Fathy, Yasser Mogahed, Mohamed El-Habiby
}

\begin{abstract}
: the demand for navigation systems is rapidly increasing, especially in GNSS-denied environments. The ubiquitous use of smart mobile devices equipped with various sensors encouraged many researchers to investigate their use in improving indoor navigation, where GNSS is not available. Inertia navigation sensors installed in mobile devices are normally low cost and drift significantly. Consequently, there is a need for auxiliary systems to aid the navigation process, which can be achieved using external sensors or additional information extracted from, for example, base maps.

In this research paper, maps have been selected as a navigation aid. Previously, maps were used for navigation aiding through geospatial data models and map-matching algorithms. These methods are based on creating geospatial data models on the fly and integrating them in the navigation database, which makes them computationally expensive and time-consuming.

In this research paper, the maps were used in an innovative way. The map directions were used in Pedestrian a dead reckoning (PDR) mode to improve the low-accuracy directions derived from portable device sensors. This method is significantly computationally efficient compared to traditional geospatial map-matching algorithms. The new approach replaces the traditional geospatial database with a list of street directions and paths that are used as Map Heading Constraints (MHC) when navigating (walking) in straight directions.
\end{abstract}

The proposed technique was tested on trajectories in GNSS denied environment (underground parking) using an iphone6s smart-phone and compared with other solutions that used the portable device sensors only. The comparison showed a significant improvement in position accuracy (up to $90 \%$ ) in comparison to using the portable device sensors only (no aiding).

Keywords: PDR, MEMS, kalman Filter, GNSS-Denied Environment, Map Heading Constraint.

\section{INTRODUCTION}

GNSS is the main navigation system in outdoor and open sky

Revised Manuscript Received on April 25, 2020.

* Correspondence Author

Mohamed Shebl*, Assistant lecturer, Public Works Department, Ain Shams University/ Faculty of Engineering/ Cairo, Egypt.

Mohamed El-Tokhey, Professors, Public Works Department, Ain Shams University/ Faculty of Engineering/ Cairo, Egypt.

Tamer Fathy, Professors, Public Works Department, Ain Shams University/ Faculty of Engineering/ Cairo, Egypt.

Yasser Mogahed, Associate professor, Public Works Department, Ain Shams University/ Faculty of Engineering/ Cairo, Egypt.

Mohamed El-Habiby, Associate professor, Public Works Department, Ain Shams University/ Faculty of Engineering/ Cairo, Egypt.

(C) The Authors. Published by Blue Eyes Intelligence Engineering and Sciences Publication (BEIESP). This is an open access article under the CC BY-NC-ND license (http://creativecommons.org/licenses/by-nc-nd/4.0/) environments, but the GNSS signals are partially blocked by buildings or totally blocked in underground environments, which makes the navigation impossible [1]. The challenge is how to keep the position's quality when the GNSS signal is lost using low-cost MEMS that built-in smart devices. Pedestrian Dead-reckoning (PDR) algorithm based on estimate the traveled distance from step counting and user stride length in addition to user heading. The main drawback of PDR is the error accumulation, especially the MEMS (gyroscopes and accelerometers) sensors drift with time.

Many navigation systems now, depend on digital maps as a tool for presenting navigation information to the end-user [2]. In this research paper, the heading constraint of paths from a base map is used to overcome the drifting problem of MEMS The PDR system uses a foot-mounted external proximity sensor for step detection in addition to Smart portable device navigation sensors, which contains a 3-axis accelerometer, 3 -axis gyroscope, and 3-axis magnetometer. In order to obtain reliable heading during navigation, the gyroscope should be integrated with a magnetometer and accelerometer though extended kalman filter (EKF). In order to improve the accuracy, the calculated heading from (EKF) was bench marked for turning to differentiate between the straight walking and other walking directions. The straight walking with the estimated heading from (EKF) is compared with the list of paths headings of a base map. The computed heading from the smart portable device sensors by (EKF) concept is replaced with the closest one from the list of estimated headings from the map. This is applied for only straight-line walking and not during turns. During turns, the heading from the EKF is used as is.

\section{SYSTEM FRAME WORK}

The system architecture is presented in figure 1, at first the sensor readings from the accelerometer, gyroscope, and magnetometer are calibrated to compensate for the deterministic errors, such as bias and scale factor. The calibration parameters for each sensor pre-determined in lab using six-position test [3] for accelerometer, and rotation test [4] for gyroscope, however magnetometer calibration done using ellipsoidal fitting [5] . Second, external hardware based on proximity sensor used for step detection and counting because it's more accurate in detection of step where it has a simple shape of measurement signal. Third, this is integrated with empirical model for user stride length estimation used for distance estimation.

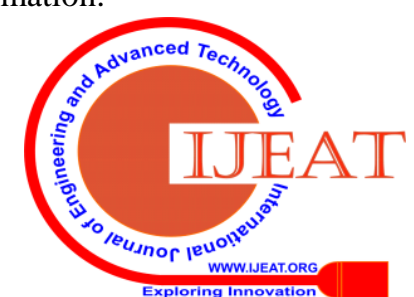


Fourth, quaternion based extended Kalman filter used for multi-sensor heading fusion to obtain stable heading at every detected step. Finally, the obtained heading checked for turning that happens during navigation and the heading is adjusted by closest pre-estimated heading in a base map that is used for aiding the navigation solution.

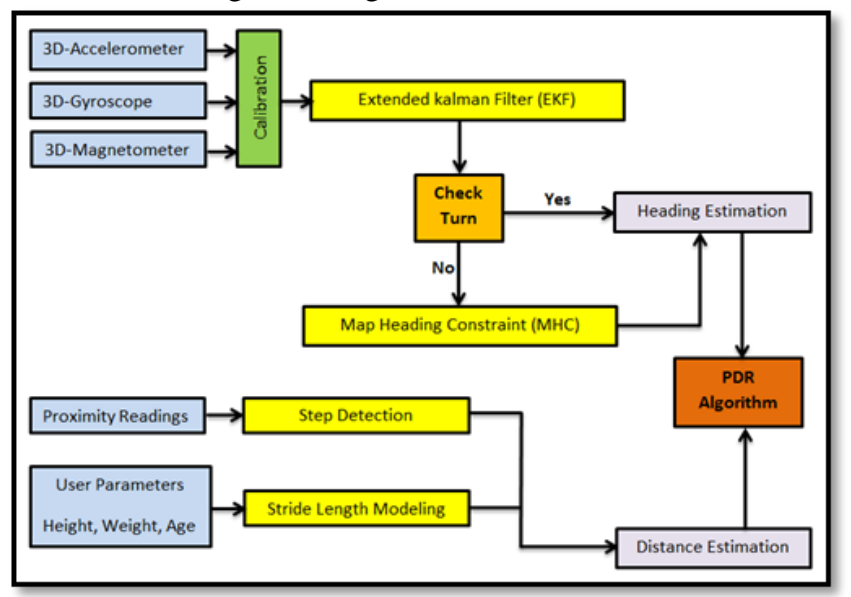

Fig. 1: PDR system architecture

\section{DISTANCE ESTIMATION}

Inertial positioning is based on the simple principle that differences in position can be determined by a double integration of acceleration. But this concept suffers from error accumulation due to mathematical integration.so the modern concept offers a new technique for inertial sensors by using the kinematic qualities of human gait [6],[7],[8],[9] and prevents the need for continuous integration processes even when the user is not moving which cause error accumulation. For step detection in walking mode, a proximity sensor (PS) is attached to one of the two feet of a person, making its face directed as close as possible to ground to ensure that the (PS) is correctly working (figure 2). The PS will counts number of steps equal to half of the number of steps of walking since the sensor attached to only one foot. The step will be sensed when the (PS) is at a position nearest to the ground where the (PS) will record (zero=off) and will be recorded as a step; otherwise, it will record the maximum range in $\mathrm{cm}$ (on) that can be detected by (PS) [10].

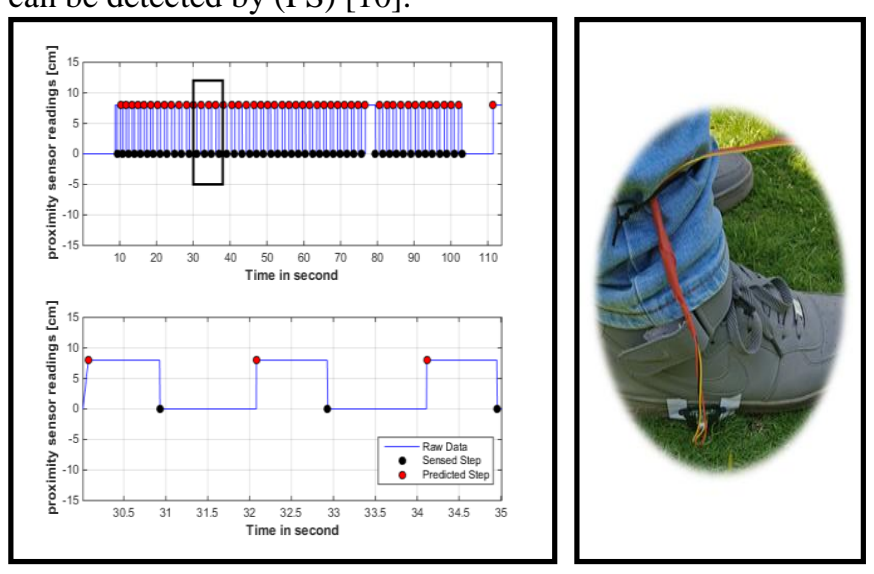

Fig. 2: PS attached to the foot of the user and its Readings Numerous studies in the forensic field were done to predict the relationship between human height and stride length. Studies have shown that the stride length divided by height is within the range of approximately 0.41 to 0.45 [11]. These studies neglect some factors such as age and weight of the person.So, a combined least squares adjustment algorithm is used to fit the input data of users (age, height, and weight) and the output (stride length) to represent an empirical equation [10].

$$
\begin{gathered}
\mathrm{SL}_{\mathrm{m}}=-4.889 * 10^{-4} * \mathrm{~A}_{\mathrm{year}}+4.3 * 10^{-3} * \mathrm{H}_{\mathrm{cm}} \\
-1.161 * 10^{-4} * \mathrm{~W}_{\mathrm{kg}}
\end{gathered}
$$

Where:

$S L$ : Stride length of the user in meters.
$A$ : Age of user in years.
$\boldsymbol{H}$ : Height of user in $\mathrm{cm}$.
$W$ : Weight of user in $\mathrm{Kg}$.

The traveled distance can be estimated by multiplying the number of detected steps with the estimated stride length of the user.

\section{HEADING ESTIMATION BY SENSOR FUSION (EKF)}

The sensor fusion method is used to estimate the device attitude by merging the measurements of 3-gyroscopes, 3accelerometers, and 3-magnetometers [12]. Gyroscopes measure orientation by integrating angular velocities while magnetometers give disturbed, but drift-free absolute orientation. However, the accelerometer measurements used for calculation of roll and pitch angles that used to transform the magnetometer's measurements from the sensor frame to the horizontal level frame.The Kalman filter (KF) building rule is using two independent models, which are the kinematic and observation. Each model has a functional and stochastic part.

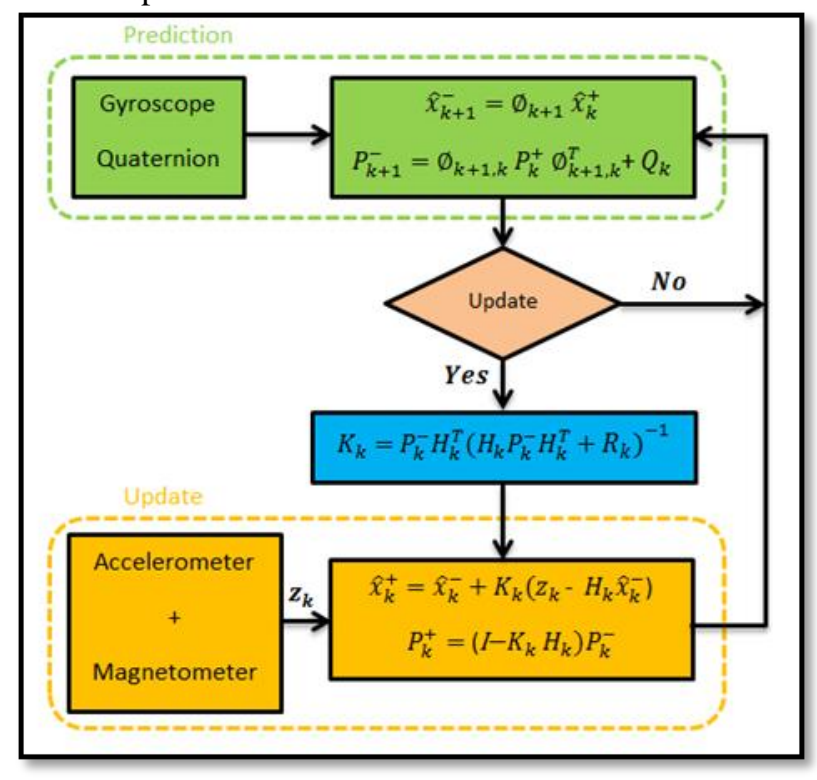

Fig. 3: KF flowchart

General KF algorithm contains two major steps: a prediction and update step [13],[14]. The prediction step reflects the influences because of the change in the states and states-covariance over time while the update step submits combined information of the states and states-covariance [12]. The strength of the KF technique lies in its ability to recursively estimate current states based on previous time steps and current measurement input data.Consequently,

Published By:

Blue Eyes Intelligence Engineering 
the principle of the presented KF algorithm is using the gyroscope measurements on the prediction step and magnetometer data in the update step.Figure 3 shows the flowchart of kalman filter procedure implemented in this research paper.

\section{A. The States Error Model}

The state vector consists of a seven-dimensional vector where the first four elements are being errors in the quaternion elements and the last three being the three-dimensional gyroscope biases. The biases in the gyroscope readings can be corrected using the readings at static intervals when the experiment starts [12].

$$
\chi=\llbracket \begin{array}{lllllll}
q_{1} & q_{2} & q_{3} & q_{4} & b_{\omega x} & b_{\omega y} & b_{\omega \Sigma}
\end{array} \rrbracket^{T}
$$

Where:

$$
\begin{aligned}
& q_{12,34}: \text { Quaternion elements } \\
& b_{w(x y z)}: \text { Gyroscope biases in three axis }
\end{aligned}
$$

\section{B. The States Transition Model}

Gyroscope 3D-angular rate measurements used for attitude estimation through the quaternion derivative are as follows:

$$
\dot{q}=\llbracket\left[\begin{array}{l}
q_{1} \\
\dot{q}_{2} \\
\dot{q_{3}} \\
\dot{q}_{4}
\end{array}\right]=\frac{1}{2} \llbracket\left[\begin{array}{cccc}
0 & -\omega_{1} & -\omega_{2} & -\omega_{3} \\
\omega_{1} & 0 & \omega_{a} & -\omega_{2} \\
\omega_{2} & -\omega_{3} & 0 & \omega_{1} \\
\omega_{3} & \omega_{2} & -\omega_{1} & 0
\end{array}\right] \llbracket\left[\begin{array}{l}
q_{1} \\
q_{2} \\
q_{3} \\
q_{4}
\end{array}\right]
$$

Where:

\section{q : Quaternion derivative}

$\omega_{1,2, a}$ : Angular rate measurements of gyroscopes in three axes.

Quaternions are used for orientation representation in the KF design because they don't suffer from the singularity problem like Euler angles [15].

\section{The measurements model}

The KF update is designed depending on the magnetic heading. The magnetic heading estimated from calibrated measurements of the magnetometer sensor that smoothed by low pass filter in addition to calibrated accelerometer measurements [16].the check for magnetic field disturbance is based on threshold that defined as a normal margin for the signal variation.

$$
Z=\llbracket q_{z 1} \quad q_{z 2} \quad q_{z a} \quad q_{z 4} \rrbracket^{T}
$$

Where:

$q_{z(1234)}:$ Quaternion elements from accelerometer and magnetometer data.

\section{EXPERIMENT AND RESULTS}

To test the performance of the proposed PDR algorithm using the map heading constraint (MHC) solution, an underground garage was selected as a GNSS-denied environment. The route started from the entrance of the garage and ended at the exit as an outdoor environment and consisted of a group of straights and turns in GNSS signal blockage environment.

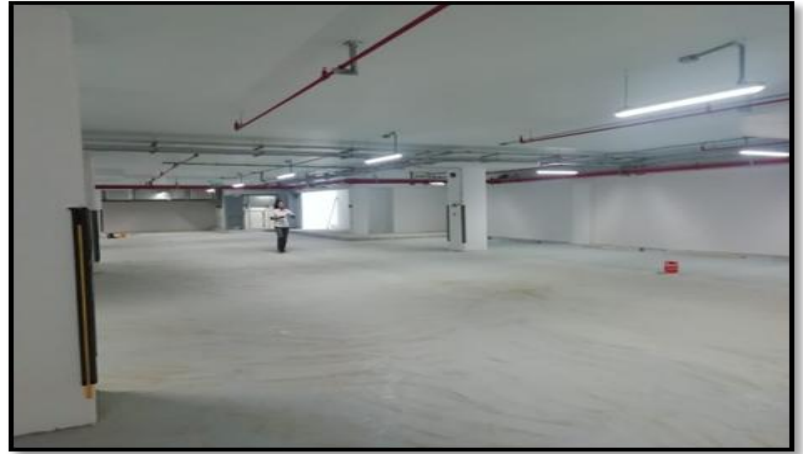

Fig. 4: PDR testing environment in an underground garage

The proposed route was surveyed with a total station and a map is produced as a surveying grade ground truth to be a reference for the proposed navigation solution.

The used smartphone is an iPhone 6s plus and the data was collected by the "sensor log" application and the measurements frequency is $100 \mathrm{~Hz}$.

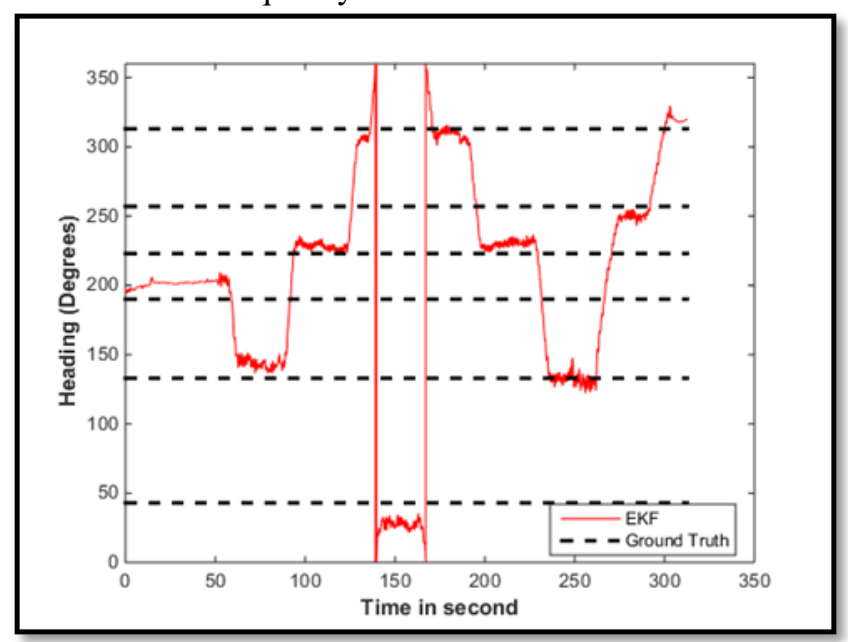

Fig. 5: Estimated Heading using EKF

First, we applied only EKF in heading estimation mode and estimated the positions of the navigated route. The results are compared to the ground truth surveyed route Huge positional error is found because of the heading estimation error, as shown in figure 5.The error drifts dramatically with extended outage periods as shown in figure 6 . The comparison is done every 10 meters between the ground truth and navigation solution.

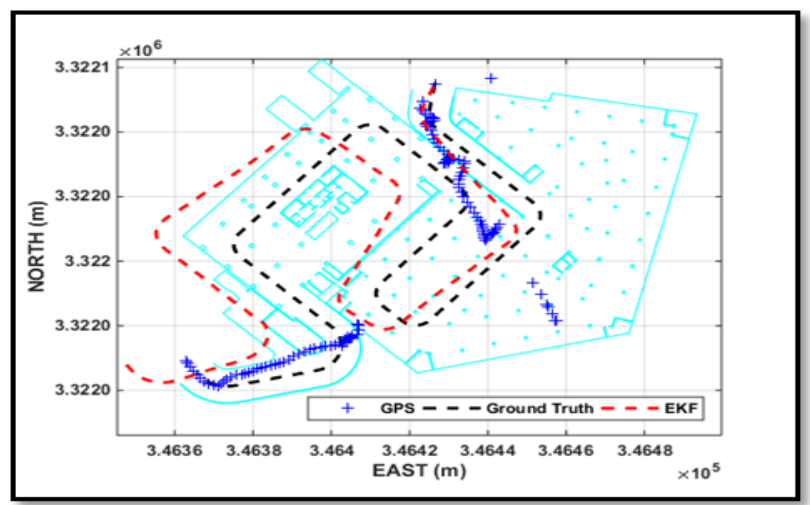

Fig. 6: PDR Navigation route by applying EKF. 
TABLE 1

Positioning Errors in navigated route based EKF compared with reference data.

\begin{tabular}{|c|c|c|c|c|c|}
\hline $\begin{array}{c}\text { Loc. } \\
\text { NO. }\end{array}$ & $\begin{array}{c}\text { Error } \\
(\mathrm{m})\end{array}$ & $\begin{array}{c}\text { Loc. } \\
\text { NO. }\end{array}$ & $\begin{array}{c}\text { Error } \\
(\mathrm{m})\end{array}$ & $\begin{array}{c}\text { Loc. } \\
\text { NO. }\end{array}$ & $\begin{array}{c}\text { Error } \\
(\mathrm{m})\end{array}$ \\
\hline 1 & 0.000 & 12 & 8.917 & 23 & 23.453 \\
\hline 2 & 2.083 & 13 & 7.988 & 24 & 22.615 \\
\hline 3 & 3.120 & 14 & 10.375 & 25 & 11.936 \\
\hline 4 & 4.839 & 15 & 13.044 & 26 & 10.936 \\
\hline 5 & 6.313 & 16 & 16.876 & 27 & 10.888 \\
\hline 6 & 7.750 & 17 & 19.978 & 28 & 11.096 \\
\hline 7 & 7.025 & 18 & 20.152 & 29 & 19.380 \\
\hline 8 & 7.383 & 19 & 21.071 & 30 & 23.834 \\
\hline 9 & 7.864 & 20 & 21.536 & 31 & 23.567 \\
\hline 10 & 8.189 & 21 & 21.926 & 32 & 23.342 \\
\hline 11 & 8.746 & 22 & 22.676 & 33 & 22.293 \\
\hline
\end{tabular}

The total route length almost 325 meters and table 1 shows the positional error at every checked location. The maximum error is 23.834 meters with RMSE equal to 15.537 meters, and relative error equal to $5.63 \%$.

To check accuracy enhancement in positioning we apply the principle of map heading constraint (MHC) and compared the same previous check location with the navigated one. Figure 7 shows the matching between the estimated heading and reference one.

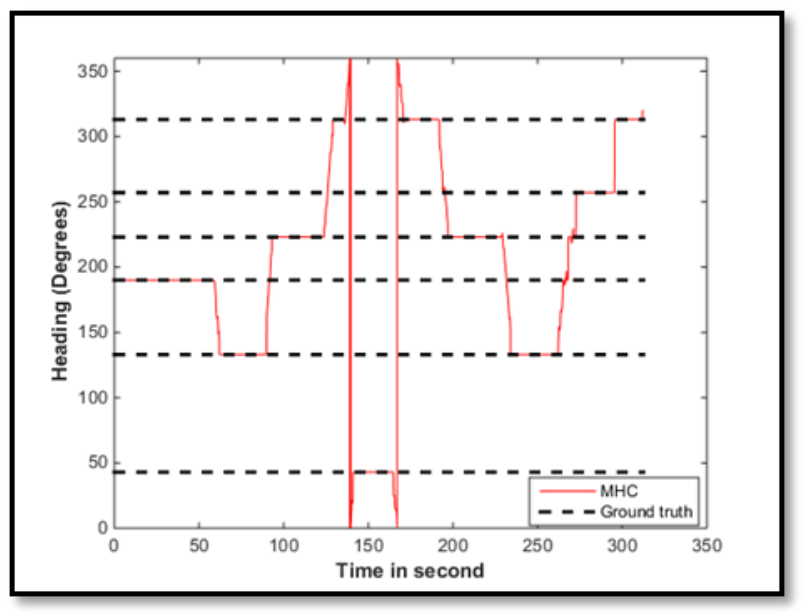

Fig. 7: Estimated Heading using MHC

The position improvement is shown in figure 8

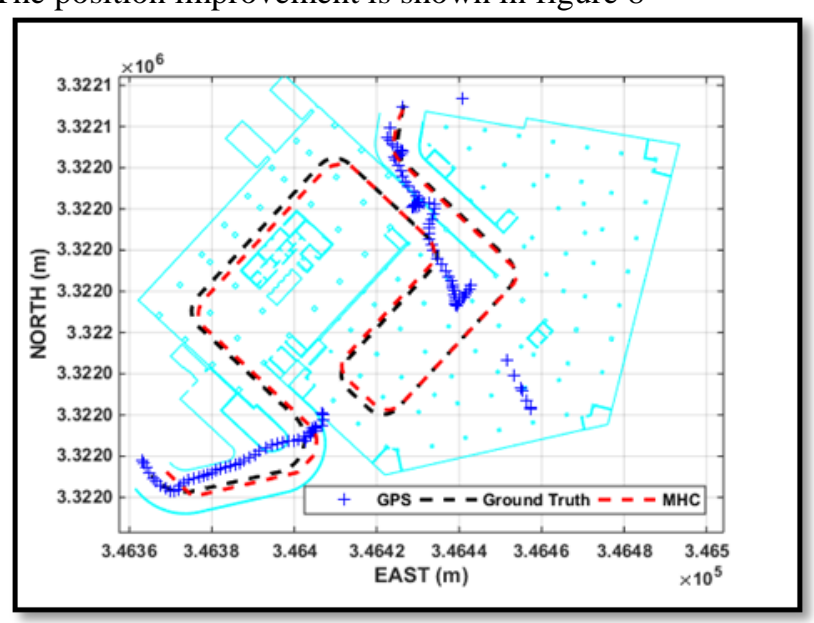

Fig. 8: PDR navigation route by applying MHC.
Table 2 presents the positional error after applying (MHC) principal. The maximum error is 2.413 meters and the RMSE is 1.292 meters, with relative error equal to $0.41 \%$. From this comparison, a significant improvement in position accuracy is achieved with up to $90 \%$.

TABLE 2

Positioning errors in navigated route-based MHC compared with reference data.

\begin{tabular}{|c|c|c|c|c|c|}
\hline $\begin{array}{c}\text { Loc. } \\
\text { NO. }\end{array}$ & $\begin{array}{c}\text { Error } \\
(\mathrm{m})\end{array}$ & $\begin{array}{c}\text { Loc. } \\
\text { NO. }\end{array}$ & $\begin{array}{c}\text { Error } \\
(\mathrm{m})\end{array}$ & $\begin{array}{c}\text { Loc. } \\
\text { NO. }\end{array}$ & $\begin{array}{c}\text { Error } \\
(\mathrm{m})\end{array}$ \\
\hline 1 & 0.000 & 12 & 2.032 & 23 & 1.381 \\
\hline 2 & 0.523 & 13 & 0.191 & 24 & 1.361 \\
\hline 3 & 0.627 & 14 & 0.875 & 25 & 1.970 \\
\hline 4 & 0.652 & 15 & 0.855 & 26 & 1.184 \\
\hline 5 & 0.677 & 16 & 1.482 & 27 & 1.224 \\
\hline 6 & 0.685 & 17 & 1.022 & 28 & 1.239 \\
\hline 7 & 0.027 & 18 & 1.030 & 29 & 2.413 \\
\hline 8 & 0.008 & 19 & 1.038 & 30 & 2.178 \\
\hline 9 & 0.044 & 20 & 1.911 & 31 & 1.817 \\
\hline 10 & 0.080 & 21 & 1.422 & 32 & 1.891 \\
\hline 11 & 1.467 & 22 & 1.402 & 33 & 1.654 \\
\hline
\end{tabular}

\section{CONCLUSION}

Navigation in most outdoor applications are guaranteed by global navigation satellite systems, but some places remain not serviced by these systems because of signal blockages such as urban canyons, as well as, underground tunnels and garages. Therefore, navigation in these circumstances will be a great challenge, especially using low-cost sensors in smart devices where error accumulation significantly drifts. In this research paper, the headings of the map routes were used for aiding and improving of the accuracy of the heading estimation using a digital map as an aid to the navigation process. A method called map headings constraint (MHC) and it was tested on underground garages implemented and used to improve indoor navigation using smart devices. Using iPhone 6s smart-phone and compared with extended kalman filter (EKF) solution. The comparison illustrated the significant improvement in position accuracy that reached to $90 \%$.

\section{REFERENCES}

1 Tian Z., Y. Zhang, M. Zhou, and Y. Liu. 2014: Pedestrian dead reckoning for MARG navigation using a smartphone. EURASIP Journal on Advances in Signal Processing, 16(1):2-11, 2014.

2 Attia M., A. Moussa, N. El-Sheimy, 2013: Map Aided Pedestrian Dead Reckoning Using Buildings Information for Indoor Navigation Applications, Positioning, 2013, 4, 227-239.

3 Syed Z. F., P Aggarwal, C Goodall, X Niu and N El-Sheimy 2007 : A new multi-position calibration method for MEMS inertial navigation systems. Meas. Sci. Technol. 18 (2007) 1897-1907

4 Marotto, V., M. Sole, T. Dessì , A. Manchinu, D. Carboni,and A. Serra 2013: Orientation Analysis through a Gyroscope Sensor for Indoor Navigation Systems.

5 Cui, X., Y. Li, Q. Wang, M. Zhang, and J. Li 2018: Three-axis magnetometer calibration based on optimal ellipsoidal fitting under constraint condition for pedestrian positioning system using foot-mounted inertial sensor/magnetometer. 2018 IEEE/ION Position, Location and Navigation Symposium (PLANS), 166-174.

6 Beauregard S. (2007): Omnidirectional pedestrian navigation for first responders. Positioning Navigation and Communication 2007, WPNC'07. 4th Workshop on, IEEE: 33-36.

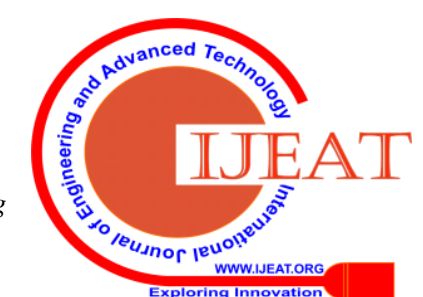


$7 \quad$ Suh Y. S., and S. Park (2009): Pedestrian inertial navigation with gait phase detection assisted zero velocity updating, Autonomous Robots and Agents, 2009. ICARA 2009. 4th International Conference on, IEEE: 336-341.

8 Lee S., K. Mase (2001): Recognition of walking behaviors for pedestrian navigation. Control Applications, 2001. (CCA'01) Proceedings of the 2001 IEEE International Conference on, IEEE: 1152-1155.

$9 \quad$ Stirling R., J. Collin, K. Fyfe and G. Lachapelle (2003): An innovative shoe-mounted pedestrian navigation system. Proceedings of European Navigation Conference GNSS: 110-115.

10 Shebl M., M. El-Tokhey, T. Fathy, Y. Mogahed, M. El-Habiby 2018: Distance measurement using proximity sensor in pedestrian and bicycle navigation, International Journal of Engineering \& Technology, 7 (4) (2018) 4266-4270.

11 Guest R., O. Miguel Hurtado, S. Stevenage, S. Black. 2017: Exploring the relationship between stride, stature and hand size for forensic assessment. Journal of Forensic and Legal Medicine, 52 (2017) 46:55

12 Ali, A., \& N. El-Sheimy, (2013). Low-Cost MEMS-Based Pedestrian Navigation Technique for GPS-Denied Areas. J. Sensors, 2013, 197090:1-197090:10.

13 Akram M. A, P. Liu, M. O. Tahir, W.Ali, Y. Wang 2019: A State Optimization Model Based on Kalman Filtering and Robust Estimation Theory for Fusion of Multi-Source Information in Highly Non-linear Systems, Sensors 2019, 19, 1687; doi:10.3390/s19071687.

14 Zeng Q, Chen W, Liu J, Wang H.2017: An Improved Multi-Sensor Fusion Navigation Algorithm Based on the Factor Graph, Sensors (Basel). 2017;17(3):641. Published 2017 Mar 21 doi:10.3390/s17030641

15 Wang L., Z. Zhang, and P. Sun 2015: Quaternion-based Kalman filter for AHRS using an adaptive-step gradient descent algorithm, Int. J. of Advanced Robotic Systems, vol. 12, no. 9, p. 131, 2015.

16 Wu D., L. Xia, J. Geng 2018: Heading Estimation for Pedestrian Dead Reckoning Based on Robust Adaptive Kalman Filtering, Sensors 2018, 18, 1970; doi:10.3390/s18061970. 